Rev Inv Vet Perú 2015; 26(1): 57-65

http://dx.doi.org/10.15381/rivep.v26i1.10917

\title{
Detección del Gen PrP de Scrapie en Ovinos Junín
}

\author{
Detection of the PrP Gene in Junin SheeP
}

Kamilo Rivera J. ${ }^{1}$, Lenin Maturrano H., ${ }^{2,3,4}$, Juan Manuel Aguilar ${ }^{3}$, Raúl Rosadio A., ${ }^{1,3}$

\section{Resumen}

El objetivo del presente trabajo fue establecer la frecuencia de genotipos de resistencia y susceptibilidad de padecer la enfermedad de scrapie en ovejas Junín. Se analizaron 56 muestras de sangre de ovinos de una unidad de producción privada de la región central del Perú. Se extrajo el ADN y los productos de PCR fueron analizados mediante digestión enzimática con endonucleasas de restricción que reconocen los polimorfismos del gen PrP. Se pudo identificar 4 de los 5 haplotipos reportados para el gen PrP: ARR (48.2\%), ARQ (35.7\%), AHQ (10.7\%) y VRQ (5.4\%). Además, se encontró 7 de los 15 genotipos reportados para el gen Prión: ARR/ARR (17.9\%), ARR/AHQ (10.7\%), ARR/ ARQ (42.9\%), ARQ/AHQ (10.7\%), ARQ/ARQ (7.1\%), ARR/VRQ (7.1\%) y ARQ/VRQ (3.6\%). El $82.1 \%$ de las ovejas presentaron genotipos de resistencia a la enfermedad y el $17.9 \%$ presentó genotipos de susceptibilidad.

Palabras clave: scrapie; gen Prión; ovino Junín

\section{Abstract}

The aim of this study was to establish the frequency of resistance and susceptible genotypes to scrapie in the Junin sheep breed. For this, 56 blood samples were collected in a sheep farm in the central highlands of Peru. The DNA was extracted and the PCR products were analyzed by enzymatic digestion using restriction endonucleases that recognize the PrP gene polymorphisms. Four of the 5 haplotypes reported for the PrP gene were identified: ARR (48.2\%), ARQ (35.7\%), AHQ (10.7\%) and VRQ (5.4\%). In

\footnotetext{
${ }^{1}$ Laboratorio de Microbiología y Parasitología Veterinaria ${ }^{2}$ Laboratorio de Zootecnia y Producción Agropecuaria, Facultad de Medicina Veterinaria, Universidad Nacional Mayor de San Marcos, Lima

${ }^{3}$ Instituto de Investigación y Desarrollo de Camélidos Sudamericanos - CONOPA, Lima

${ }^{4}$ E-mail: lenin.maturrano@gmail.com
}

Recibido: 26 de marzo de 2014

Aceptado para publicación: 4 de setiembre de 2014 
addition, 7 of the 15 reported genotypes for the Prion gene were found: ARR/ARR(17.9\%), ARR/AHQ (10.7\%), ARR/ARQ (42.9\%), ARQ/AHQ (10.7\%), ARQ/ARQ (7.1\%), ARR/ VRQ $(7.1 \%)$ and ARQ/VRQ (3.6\%). Finally, $82.1 \%$ of the animals had genotypes resistant to the disease and $17.9 \%$ showed genotypes of susceptibility.

Key words: scrapie; Prion gene; Junin sheep

\section{INTRODUCCIÓN}

La tembladera o scrapie es una encefalopatía espongiforme transmisible (EET) que afecta a ovejas y cabras. Fue descrita por primera vez en 1732 (Detwiler y Baylis, 2003), y ha recibido especial atención en años recientes, debido a la alarma social generada por la posible transmisión al hombre desde el bovino. La sintomatología incluye prurito, hiperexcitabilidad, temblores y falta de coordinación en la marcha, para acabar en parálisis y muerte. La enfermedad presenta largos periodos de incubación y da lugar a la degeneración progresiva del sistema nervioso central que, en etapas avanzadas de la enfermedad, adquiere el característico aspecto espongiforme (Portela et al., 2006).

El nombre de prión deriva de proteína infecciosa (Prusiner, 1995). Los priones son glucoproteínas de membrana que se anclan a ella por una molécula de glicosil-fosfatidilinositol y cumplen funciones biológicas que están relacionadas con la sinapsis (Brown, 2001) y con rutas de transducción de señales de membrana relacionadas con la apoptosis (Thellung et al., 2000). En el ovino se ha establecido la influencia del gen Prión (PrP o PRNP), en la capacidad de resistir o padecer la enfermedad. Los cambios de bases que se producen en tres codones (posiciones 136, 154 y 171) de la proteína prión van a determinar, en mayor o menor medida, la susceptibilidad de cada animal de padecer dicha enfermedad.
Se han establecido cinco grupos de riesgo de contraer la enfermedad con base al genotipo de cada animal, donde el grupo 1 incluye animales catalogados como muy resistentes o de muy bajo riesgo de contraer la enfermedad, y en el grupo 5 se incluyen los animales muy sensibles o de alto riesgo (Dawson et al., 1998). La variación que presentan los cinco haplotipos denominados ARQ, VRQ, AHQ, ARR y ARH establecen que, en general, los animales ARR/ARR son los más resistentes y los VRQ/VRQ son los más sensibles a padecer la enfermedad (Dawson et al., 1998).

En Francia (Francois et al., 2002), Islandia (Thorgeirdottir et al., 1999), Inglaterra (McIntyre et al., 2009), Bulgaria (Sirakov et al., 2011), Pakistán (Babar et al., 2009), Alemania (Lühken et al., 2004) y Estados Unidos de Norteamérica (Thompson y Gasser, 2008) se ha evaluado la frecuencia de los tipos genéticos del gen Prión en diversas razas de ovinos presentes en sus respectivas áreas geográficas. En la Unión Europea se promueven programas de erradicación de la enfermedad en ovinos, siendo un requisito realizar el genotipado de los animales que ingresan o salen de los países miembros (Comisión Europea, 2003).

En el Perú se tiene cerca de 10 millones de ovinos, entre carneros y corderos (INEI, 2012), los que representan un recurso importante de subsistencia en las regiones alto andinas del país. El grupo racial con mayor número de inividuos es el tipo criollo $(81.0 \%)$, seguido de las razas Corriedale (11.4\%), 
Hampshire Down (2.6\%), Black Belly $(0.9 \%)$ y otras razas $(4.1 \%)$ en la que se incluye a la raza Junín (INEI, 2012). El ovino Junín es considerado como la primera raza desarrollada en la región andina y una de las pocas razas creadas en el hemisferio occidental durante el siglo pasado. Se formó por selección masiva dentro de un gran rebaño cerrado, como animal adaptado a las condiciones ambientales de sierra alta y puna. El ovino Junín produce carne y lana, es fértil y de crecimiento acelerado, criado a campo abierto en pasturas naturales (Villareal y Gamarra, 1978).

El objetivo del presente trabajo fue determinar los genotipos del gen Prión en ovinos Junín, con la finalidad de establecer la frecuencia de genotipos de resistencia o susceptibilidad de padecer la enfermedad de scrapie.

\section{Materiales y Métodos}

\section{Toma de Muestras y Procesamiento}

Se recolectaron 56 muestras de sangre de ovejas mayores de 2 años, provenientes de un núcleo de crianza privada. Las muestras de sangre fueron colectadas mediante punción de la vena yugular, empleando tubos vacutainer ${ }^{\circledR}$ de $3 \mathrm{ml}$ con $\mathrm{K}_{-}$EDTA al $15 \%$. Las muestras fueron conservadas a $-20{ }^{\circ} \mathrm{C}$ y transportadas al Laboratorio de Biología y Genética Molecular de la Facultad de Medicina Veterinaria, Universidad Nacional Mayor de San Marcos (UNMSM), Lima.

\section{Extracción de ADN}

Para la extracción de ADN se utilizó el kit comercial DNeasy® Blood \& Tissue Kit (QIAGEN®), siguiendo las instrucciones del fabricante. La concentración y la tasa de pureza (relación entre la concentración de ácidos nucleicos y concentración de proteínas) de las muestras se determinó mediante espectrofotometría a 260 y $280 \mathrm{~nm}$. También se evaluó su calidad mediante electroforesis en un gel de agarosa, con un marcador de ADN de concentración conocida (ADN del fago $\lambda$ cortados con la enzima Hind III).

\section{Amplificación del Gen PrP mediante PCR}

Con la PCR (reacción en cadena de la polimerasa) se obtuvo un gran número de copias de un fragmento de ADN; en este caso, una región de aproximadamente $300 \mathrm{pb}$ del gen Prión, que incluye las posiciones 136, 154 y 171. Para ello, se prepararon mezclas de reacción con $7.5 \mu 1$ de Mix Multiplex 2X, $4.5 \mu l$ de agua ultrapura, $0.75 \mu 1$ de cada primer (F y R) y $1.5 \mu 1$ de ADN de ovino. Las condiciones de amplificación fueron: $95{ }^{\circ} \mathrm{C}$ durante 5 min, continuando con 30 ciclos a $95^{\circ} \mathrm{C}$ durante $30 \mathrm{~s}, 63{ }^{\circ} \mathrm{C}$ durante 30 s y $72{ }^{\circ} \mathrm{C}$ durante $1 \mathrm{~min}$, finalizando con un ciclo de 72 ${ }^{\circ} \mathrm{C}$ durante $10 \mathrm{~min}$. Se confirmó la presencia del gen PrP haciendo una electroforesis en gel agarosa al $2 \%$.

\section{Determinación de Genotipos del Gen PrP}

Los productos de PCR fueron analizados mediante digestión enzimática con endonucleasas de restricción que reconocen los polimorfismos del gen PrP en estudio. Para determinar los polimorfismos de los codones 136 y 154, el producto de la PCR es digerido con la enzima $B s p H I$ (T $\downarrow$ CATAG) a $37{ }^{\circ} \mathrm{C}$ durante $2 \mathrm{~h}$, como en el protocolo de Hunter (1997). En el caso del codón 136, si la enzima realiza un corte en el fragmento de ADN se tipifica como alelo $\mathrm{V}$ y si no realiza el corte se tipifica como A. Para el codón 154, si la enzima $B s p \mathrm{HI}$ corta el producto de PCR en esta posición se tipifica como alelo $\mathrm{H}$ y si no realiza el corte se tipifica como $\mathrm{R}$.

Los polimorfismos para el codón 171 se determinan según Yuzbasiyan-Gurkan et al. (1999), mediante la combinación de los resultados obtenidos por la digestión del producto de la PCR con la enzima $B s l$ I (CCNNNNN $\downarrow$ NNGG) a $55^{\circ} \mathrm{C}$, durante $2 \mathrm{~h}$ 


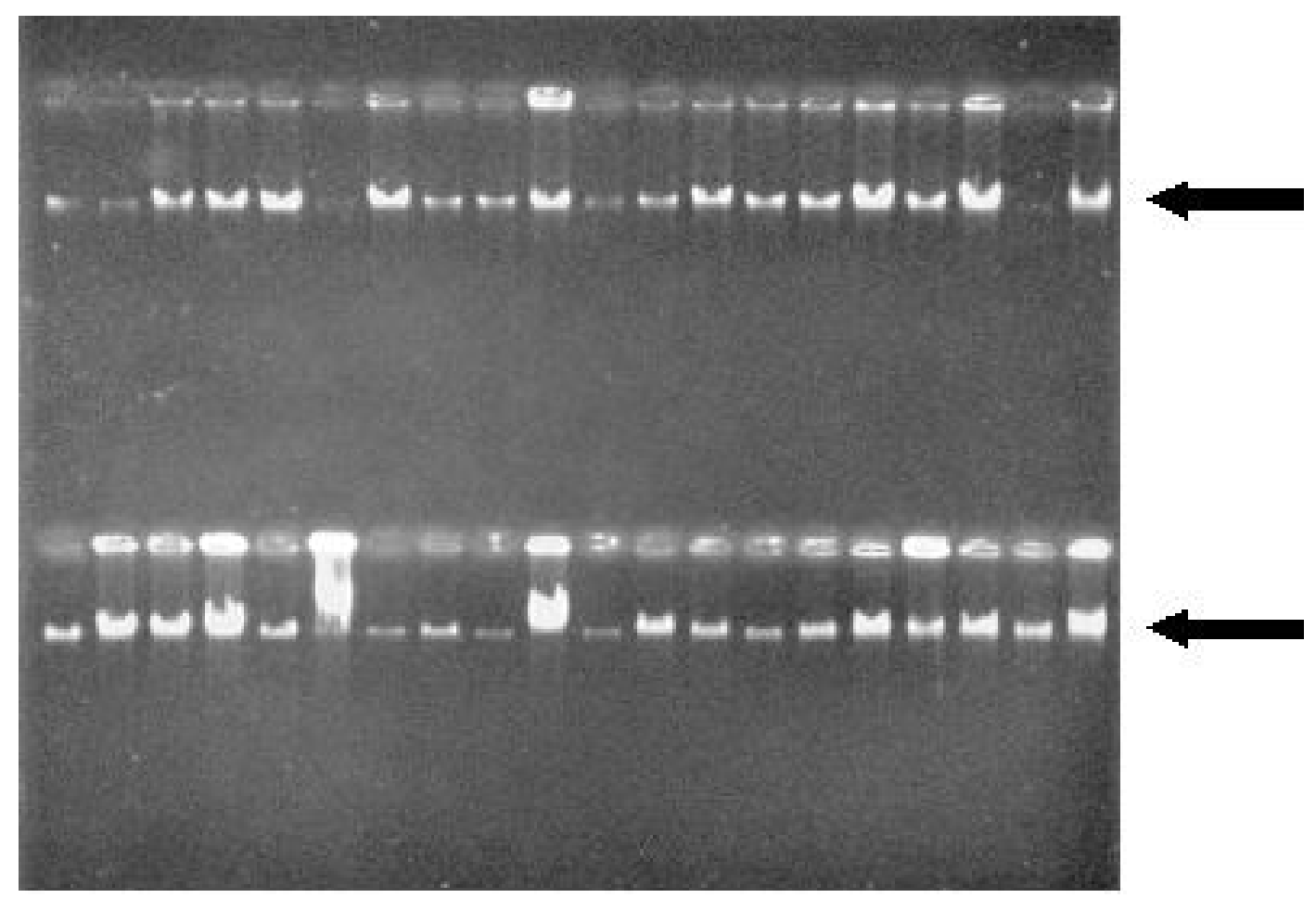

Figura 1. Gel de agarosa al 1\% mostrando las bandas de ADN obtenidas de la extracción a partir de muestras de sangre en ovinos Junín

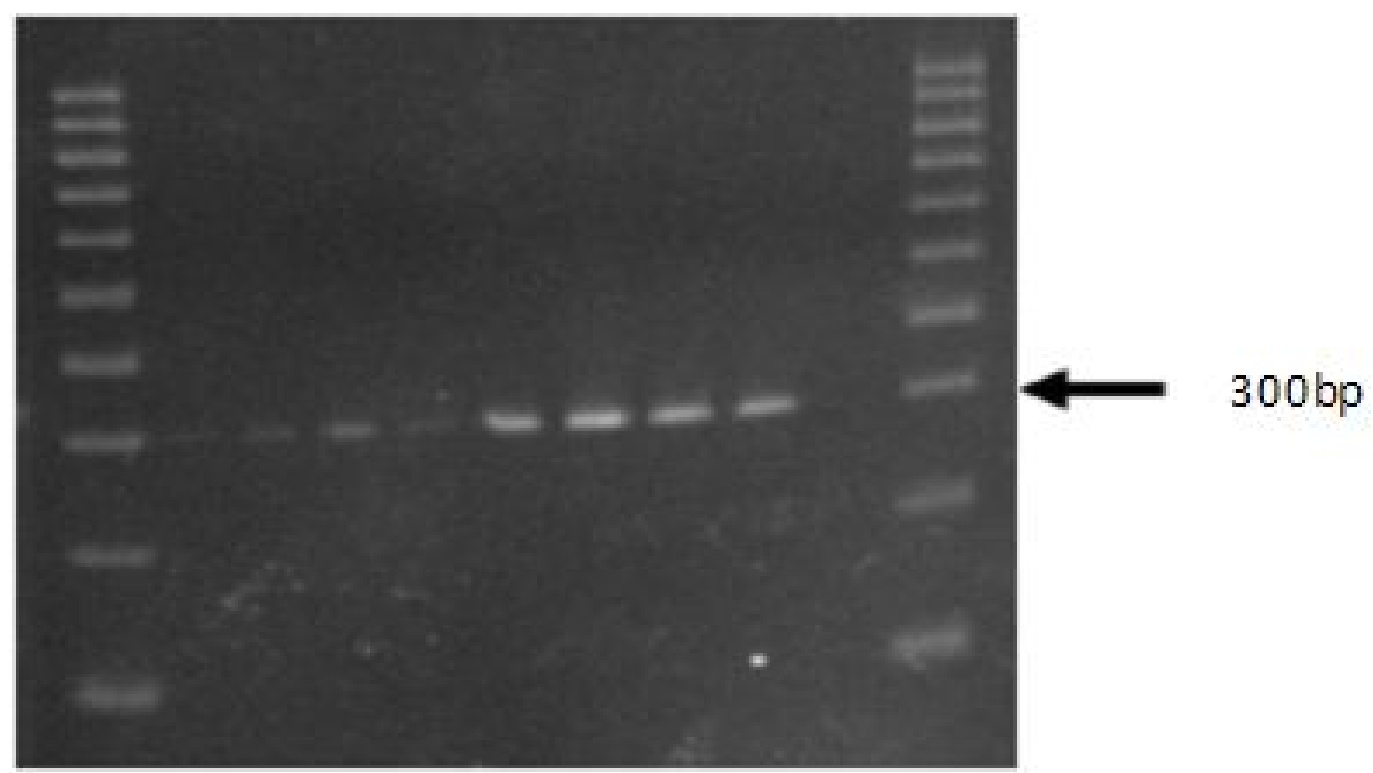

Figura 2. Amplificación del gen $\operatorname{Pr} P$ 


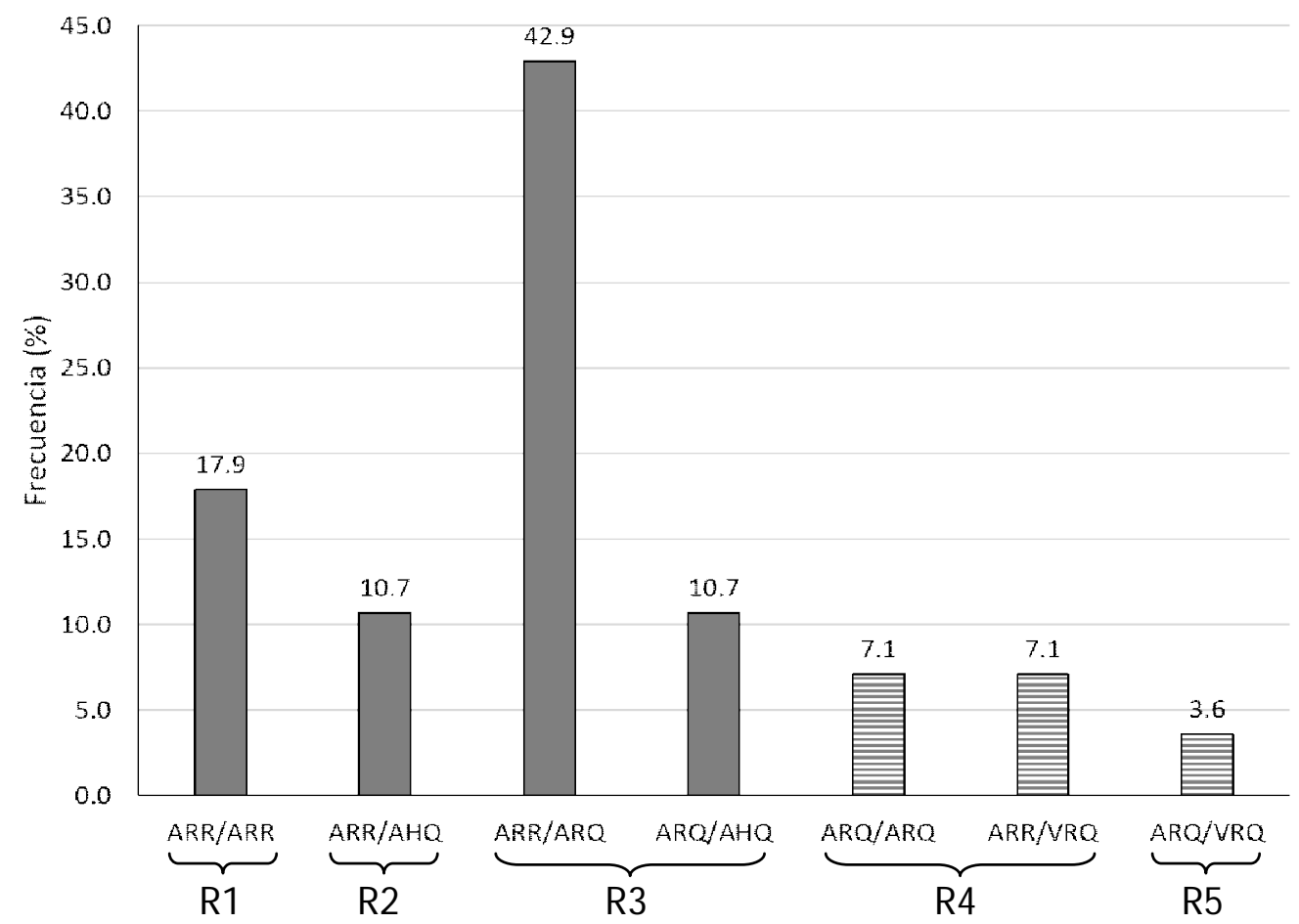

Figura 3. Proporción de genotipos y grupos de riesgo de acuerdo a las combinaciones de importancia en la enfermedad de scrapie. R1, R2 y R3 son genotipos resistentes y R4 y R5 son genotipos susceptibles

y del producto de la PCR con AccI (GT $\downarrow$ MKAC) a $37{ }^{\circ} \mathrm{C}$ durante $2 \mathrm{~h}$. Si la enzima $B s l \mathrm{I}$ corta, se le tipifica como alelo R, en caso contrario podría ser alelo Q o H. Para diferenciar los alelos Q y H se emplea la enzima AccI que al realizar corte se tipifica como alelo $\mathrm{Q}$, en caso contrario como alelo $\mathrm{H}$. Los fragmentos de restricción generados son separados por electroforesis en un gel de agarosa al 3\%. La detección de los fragmentos se realiza por tinción con bromuro de etidio $(0.5 \mu \mathrm{g} / \mathrm{mg})$ y fotografiado bajo luz UV.

\section{Resultados}

La Fig. 1 muestra el ADN en las muestras de sangre, obteniéndose concentraciones de ADN de 25 a 100 ng. Se logró amplificar el gen PrP de todas las muestras de sangre. El tamaño esperado del producto de PCR fue de $300 \mathrm{pb}$ aproximadamente (Fig. 2).

Se encontraron cuatro haplotipos para el gen Prión, siendo el de mayor frecuencia el ARR (48.2\%) y el de menor frecuencia el VRQ (5.4\%). La frecuencia para los AHQ y ARQ fue de 10.7 y $35.7 \%$, respectivamente.

Asimismo, se encontraron 7 genotipos, que, de acuerdo a los grupos de resistencia, 4 pertenecieron a genotipos de resistencia y 3 a genotipos de susceptibilidad (Fig. 3). Así, el $17.9 \%$ perteneció al grupo de resistencia R1, $10.7 \%$ al grupo R2 y $53.6 \%$ al grupo R3. En los grupos de susceptibilidad, el $14.2 \%$ perteneció al grupo R4 y el 3.6\% al grupo R5. 
Finalmente, se encontró que el $82.1 \%$ de los animales mostraron algún genotipo de resistencia, en tanto que $17.9 \%$ de las ovejas presentó un genotipo de susceptibilidad.

\section{Discusión}

La sintomatología del scrapie incluye signos de rápida identificación; sin embargo, no se ha reportado en forma fehaciente la presencia de esta enfermedad en ovinos en el Perú, aunque tampoco se puede descartar su presencia (R. Rosadio, Lima, comunicación personal). Se conoce que la proteína Prión resiste temperaturas extremas, compuestos orgánicos y otros que normalmente son capaces de desnaturalizar o destruir las proteínas (Arancibia et al., 2004). Además, se ha demostrado que la proteína infecciosa puede transmitirse a través de contacto con tejidos infectados, secreciones, e incluso por exposición a aerosoles liberados por animales infectados (Haybaeck et al., 2011).

El presente estudio permitió identificar 4 de los 5 haplotipos reportados a nivel mundial (Belt et al., 1995) y 7 de los 15 genotipos identificados para el gen Prión (Dawson et al., 1998). ARR fue el haplotipo más frecuente $(48.2 \%)$, seguido del haplotipo ARQ (35.7\%). Ianella et al. (2012) encontraron los mismos haplotipos y en el mismo orden de frecuencia, cuando evaluaron los haplotipos y genotipos en 13 razas de ovinos de Brasil, siendo uno de los pocos estudios realizados a nivel de Sudamérica. Los haplotipos ARR y ARQ son considerados de resistencia y AHQ de resistencia intermedia, mientras que VRQ es un haplotipo de susceptibilidad para el scrapie (Baylis y Goldmann, 2004).

En relación con los 7 genotipos encontrados (Fig. 3), 4 de ellos (ARR/ARR, ARR/ AHQ, ARR/ARQ, ARQ/AHQ) son considerados genotipos de resistencia y ARQ/ ARQ, ARR/VRQ y ARQ/VRQ son considerados genotipos de susceptibilidad (Dawson et al., 1998). De esta forma, el
$82.1 \%$ de las ovejas fueron consideradas como resistentes a scrapie, en tanto que el $17.9 \%$ fue considerado como susceptible.

Aunque estos valores solo corresponden a un grupo de 56 muestras, puede servir de punto de partida para futuras investigaciones, así como establecer un programa de prevención seleccionando animales resistentes a la enfermedad. El programa de selección en EEUU ha permitido disminuir los casos positivos a scrapie de $0.12 \%$ a $0.02 \%$ en algunos rebaños (American Sheep Industry Association, 2013). A nivel de América del Sur, Brasil ha reportado casos de scrapie o enfermedades causadas por priones desde 1978 (Ianella et al., 2012). Si bien se desconoce el total de casos reportados, es evidente que la proteína infecciosa está circulando en América del Sur y, por lo tanto, puede aparecer en el país. Brasil ha realizado el genotipado de 1400 ovejas de 13 razas (Ianella et al., 2012) y Uruguay (Artigas et al., 2011) viene realizando algunas pruebas, siendo los únicos estudios de este tipo en esta parte del continente.

La posibilidad de implementar estos análisis en un programa de selección facilitaría una rápida selección de animales con genotipos de alta resistencia a la enfermedad. Inclusive posibilitaría el análisis y trasferencia de embriones a madres con genotipo susceptible, pues fetos portadores del alelo ARR (resistencia a priones) que desarrollen en madres sucesptibles o que lleguen a desarrollar enfermedad pueden resistir y no acumular la proteína infecciosa (Wisniewski et al., 1996; Andreoletti et al., 2002). Por otro lado, los trabajos realizados en la selección de ovinos resistentes a scrapie concluyen que no han tenido efecto negativo contra características productivas deseadas como la producción de carne o lana (Moussaoui et al., 2005; Vitezika et al., 2006; Evoniuk et al., 2007; Sweeney y Hanrahan, 2008; Kastelic y Kompan, 2009; Boulton et al., 2010). 
puede infectar al hombre y generarle una nueva EET, pero se conoce la capacidad de adaptación y transmisión de los priones infecciosos (PrPsc) de los ovinos al ganado vacuno (Bradleyy Wilsmith, 1993; Brown et al., 2001) y finalmente al hombre, produciendo la nueva variante de Creuzfeldt Jakob (Bruce et al., 1997); por ello la necesidad de identificar los genotipos de las ovejas y orientar la selección de genotipos de resistencia. Debido a esto, se reconoce el alto riesgo de transmisión de priones infecciosos interespecie y la necesidad de implementar medidas preventivas para evitar la transmisión al hombre. La Unión Europea, EEUU y otros países (Comisión Europea, 2003) han desarrollado planes de erradicación de scrapie, a través de la identificación, selección/eliminación de ovinos resistentes/susceptibles de padecer la enfermedad.

El ovino Junín fue desarrollado con las razas Columbia, Corriedale Americano, $\mathrm{Pa}$ namá y Warhill (Villareal y Gamarra, 1978), pero se desconoce los genotipos que podrían estar presentes en estas razas. Tampoco se conoce los genotipos que podrían estar presentes en otras razas en el medio como Black Belly, Corriedale, Hampshire Down, y en el ovino criollo, de allí que se hace necesario realizar estudios adicionales en estas razas.

El presente estudio constituye el primer reporte de la identificación de haplotipos y genotipos de resistencia/susceptibilidad de scrapie en ovinos en el país.

\section{Conclusiones}

- Se encontraron 4 de los 5 haplotipos reportados (ARR, ARQ, AHQ y VRQ), y se encontraron 7 genotipos, de los cuales 4 fueron de resistencia y 3 de susceptibilidad al scrapie.

- $\quad$ El $82.1 \%$ de las muestras correspondió a un genotipo de resistencia a scrapie, en tanto que el $17.95 \%$ correspondió a un genotipo de susceptibilidad.
- El haplotipo ARR y el genotipo ARR/ ARQ fueron los más frecuentes.

\section{Agradecimientos}

Los autores agradecen al Consejo Nacional de Ciencia y Tecnología CONCYTEC (Contrato 306-2009CONCYTEC-OAJ) y al Vicerrectorado de Investigación de la UNMSM por el financiamiento del estudio.

\section{Literatura Citada}

1. American Sheep Industry Association. 2013. EEUU. [Internet], [02 setiembre 2014]. Disponible en: http:// www.sheepusa.org/

2. Andreoletti O, Lacroux C, Chabert A, Monnereau L, Tabouret G, Lantier F, Berthon P, et al. 2002. $\operatorname{Pr} \mathrm{P}(\mathrm{Sc})$ accumulation in placentas of ewes exposed to natural scrapie: influence of foetal PrP genotype and effect on eweto-lamb transmission. J Gen Virol 83: 2607-2616.

3. Arancibia M, Nicklas L, Bravo JC. 2004. Enfermedades por priones. Rev Otorrinolaringol Cir Cabeza Cuello 64: 141-148.

4. Artigas R, Passos D, Hepp D, Weimer T, Postiglioni A. 2011. Polimorfismos de los codones 136 y 171 del gen $\operatorname{PrP}$ en una majada de ovino criollo del Uruguay. Arch Zootec 60: 817-820. doi: 10.4321/ S0004-05922011000300070

5. Babar M, Farid A, Benkel B, Ahmad J, Nadeem A, Imran M. 2009. Frequencies of $\mathrm{PrP}$ genotypes and their implication for breeding against scrapie susceptibility in nine Pakistani sheep breeds. Mol Biol Rep 36: 561-565. doi: 10.1007/s11033-008-9214-7

6. Baylis M, Goldmann W. 2004. The genetics of scrapie in sheep and goats. Curr Mol Med 4: 385-396. 
7. Belt P, Muileman I, Schreuder B, Bosde Ruijter J, Gielkens A, Smits M. 1995. Identification of five allelic variants of the sheep PrP gene and their association with natural scrapie. J Gen Virol 76: 509-517.

8. Boulton K, Moore R, Bishop S. 2010. Associations of PrP genotype with lamb production traits in four commercial breeds of British upland and crossing sheep. Livestock Sci 7: 155-163. doi: 10.1016/j.livsci.2009.09.007

9. Bradley R, Wilesmith JW. 1993. Epidemiology and control of bovine spongiform encephalopathy (BSE). Br Med Bull 49: 932-959.

10. Brown P, Will RG, Bradley R, Asher DM, Detwiler L. 2001. Bovine spongiform encephalopathy and variant Creutzfeld-Jacob disease: background, evolution, and current concerns. Emerg Infect Dis 7: 6-16.

11. Brown DR. 2001. Prion and prejudice: normal protein and the synapse. Trends Neurosci 24: 85-90. doi: 10.1016/S01662236(00)01689-1

12. Bruce ME, Will RG, Ironside JW, McConnell I, Drummond D, Suttie A, McCardle L, et al., 1997. Transmissions to mice indicate that 'new variant' CJD is caused by the BSE agent. Nature 389: 498-501. doi: 10.1038/39057

13. Comisión Europea. 2003. Official Journal of the European Union [Internet], [20 agosto 2011]. Disponible en: http:// www.save-foundation.net/intern/pdf/ EU\% 20 breeding $\% 20$ progra m\% 20030213.pdf

14. Dawson M, Hoinville LJ, Hosie BB, Hunter N. 1998. Guidance on the use of PrP genotyping as an aid to the control of clinical scrapie. Vet Rec 142: 623625.

15. Detwiler L, Baylis M. 2003. The epidemiology of scrapie. Rev Sci Tech 22: 121-143.

16. Evoniuk JM, Berg PT, Johnson ML, Larson DM, Maddock TD, Stolte-now CL, Schauer CS, et al. 2007. Associations between genotypes at codon 171 and 136 of the prion protein gene and production traits in market lambs.Am J Vet Res 68: 1073-1078. doi: 10.2460/ajvr.68.10.1073

17. Francois D, Elsen J, Barillet F, Lajous D, Eychenne F, Palhiere. 2002. Breeding sheep for scrapie resistance. In: Gabiña D, Sanna S (eds). Breeding programmes for improving the quality and safety of products. New traits, tools, rules and organization? Zaragoza, España; CIHEAM. p 29-35.

18. Haybaeck J, Heikenwalder M, Klevenz B, Schwarz P, Margalith I, Bridel C, Mertz K, et al. 2011. Aerosols transmit prions to immunocompetent and immunodeficient mice. PLOS Pathogens 7: e101257. doi: 10.1371/journal. ppat.1001257

19. Hunter N. 1997. PrP genetics in sheep and the implications for scrapie and BSE. Trends Microbiol 5: 331-334. doi: 10.1016/S0966-842X(97)01081-0

20. Ianella P, McManus CM, Caetano AR, Paiva SR. 2012. PRNP haplotype and genotype frequencies in Brazilian sheep: issues for conservation and breeding programs. Res Vet Sci 93: 219-225. doi: 10.1016/j.rvsc.2011.06.025

21. INEI [Instituto Nacional de Estadística e Informática]. 2012. Lima: IV Censo Nacional Agropecuario. [Internet], [22 agosto 2013]. Disponible en: http://proyectos.inei.gob.pe/web/ D o c u m e n o s P u b l c os / ResultadosFinalesIVCENAGRO.pdf

22. Kastelic M, Kompan D. 2009. The effect of PRP genotype on growth of rams on test station. Biotechnol Anim Husbandry 25: 277-284.

23. Lühken G, Buschmann A, Groschup M, Erhardt G. 2004. Prion protein allele $\mathrm{A}_{136} \mathrm{H}_{154} \mathrm{Q}_{171}$ is associated with high susceptibility to scrapie in purebred and crossbred German Merinoland sheep. Arch Virol 149: 1571-1580. doi: 10.1007/ s00705-004-0303-1

24. McIntyre KM, Trewby H, Gubbins S, Baylis M. 2009. The impact of sheep on the risk of classical scrapie. Epidemiol 
Infect 138: 384-392. doi: 10.1017/ S0950268809990537

25. Moussaoui Y, Caja G, Casellas J, Such X, Francino O. 2005. Asociaciones entre genotipo PrP y caracteres productivos en ovino lechero de raza Manchega y Lacaune: primeros resultados en prolificidad y peso al nacimiento. En: XI Jornadas sobre Producción Animal. Zaragoza, España: Asociación Interprofesional para el Desarrollo Agrario (AIDA).

26. Portela C, Bouzada J, Prado C, Areán $H$, Fernández $M$, López $M$, Fernández A, Viana J. 2006. Genotipado del scrapie ovino: adaptación del método de análisis para la identificación de nuevas variantes alélicas. En: XIV Congreso Internacional de la Federación Mediterránea de Sanidad y Producción de Rumiantes. Santiago de Compostela, España.

27. Prusiner SB. 1995. The prion diseases. Sci Am 272: 48-56.

28. Sirakov I, Peshev R, Christova L. 2011. Genetic predisposition of some Bulgarian sheep breeds to scrapie disease. Virus Genes 43: 153-159.

29. Sweeney T, Hanrahan JP. 2008. The evidence of associations between prion protein genotype and production, reproduction, and health traits in sheep. Vet Res 39: 28. doi: 10.1051/ vetres:2008004

30. Thellung S, Florio T, Villa V, Corsaro A, Arena S, Amico C, Robello $M$, et al. 2000. Apoptotic cell death and impairment of L-type voltage-sensitive calcium channel activity in rat cerebellar granule cells treated with the prion protein fragment 106-126. Neurobiol Dis 7:299-309.

31. Thompson MK, Gasser CL. 2008. Genotypic variation at the prion protein gene among Suffolk, Rambouillet, and Targhee sheep. Proc Am Soc Anim Sci 58: 72-75.

32. Thorgeirdottir S, Sigurdarson S, Thorisson H, Georgsson G Palsdottir A. 1999. PrP gene polymorphism and natural scrapie in Icelandic sheep. J Gen Virol 80: 2527-2534.

33. Villareal J, Gamarra M. 1978. El ovino raza Junín. Sociedad Agrícola de Interés Social Túpac Amaru 1: 3-9.

34. Vitezica Z, Moreno CR, Bodin $L$, François D, Barillet F, Brunel JC, Elsen JM. 2006. No associations between PrP genotypes and reproduction traits in INRA 401 sheep. J Anim Sci 86: 1317-1322.

35. Wisniewski HM, Sigurdarson S, Rubenstein R, Kascsak RJ, Carp RI. 1996. Mites as vectors for scrapie. Lancet 347: 1114. doi:10.1016/S01406736(96)90310-4

36. Yuzbasiyan-Gurkan V, Krehbiel JD, Cao Y, Venta PJ. 1999. Development and usefulness of new polymerase chain reaction-based tests for detection of different alleles at codons 136 and 171 of the ovine prion protein gene. Am $\mathrm{J}$ Vet Res 60: 884-887. 